\title{
LEUCOPLASIA BUCAL - QUE LESÃO É ESTA?
}

Felipe Vinícius Rosa Ruiz, Gisele Alborghetti Nai

Universidade do Oeste Paulista - UNOESTE, Faculdade de Odontologia, Departamento de Patologia, Presidente Prudente, SP. E-mail: patologia@unoeste.br

\section{RESUMO}

O câncer da cavidade bucal é um dos dez cânceres mais frequentes ao redor do mundo. O carcinoma espinocelular (CEC) da boca e da cabeça e pescoço é a forma mais comum de câncer e está associado à presença de lesões pré-neoplásicas. A leucoplasia bucal é definida como uma placa branca que não pode ser caracterizada clinicamente ou patologicamente. O objetivo desta revisão é abordar os diagnósticos diferenciais anatomo-patológicos da leucoplasia bucal. Foi realizada uma revisão bibliográfica narrativa, com levantamento de artigos científicos sobre leucoplasia bucal, até o ano de 2015. Aproximadamente, $3 \%$ da população mundial tem leucoplasia. Destas, 5 a 25\% são lesões pré-neoplásicas. Microscopicamente, a leucoplasia pode ser caracterizada por uma gama de alterações, como hiperplasia de células escamosas, displasia epitelial, carcinoma in situ e CEC invasor. No caso das leucoplasias bucais ainda dependemos fortemente do julgamento clínico, biópsias adequadas e do exame histopatológico para definição do seu potencial de malignização.

Palavras-chave: leucoplasia bucal, neoplasias bucais, patologia, diagnóstico, biópsia.

\section{ORAL LEUKOPLAKIA - WHAT INJURY IS THAT?}

\begin{abstract}
Cancer of the oral cavity is one of the ten most common cancers worldwide. Squamous cell carcinoma (SCC) of the mouth and head and neck is the most common form of cancer and is associated with the presence of precancerous lesions. The oral leukoplakia is defined as a white plate which can not be characterized clinically or pathologically. The aim of this review is addressing the histopathological differential diagnosis of oral leukoplakia. A narrative literature review was conducted a survey of scientific articles on oral leukoplakia, by the year 2015. Approximately 3\% of the world population has leukoplakia. Of these, 5 to $25 \%$ are preneoplastic lesions. Microscopically, leukoplakia can be characterized by a range of changes, such as squamous cell hyperplasia, epithelial dysplasia, carcinoma in situ and invasive SCC. In the case of oral leukoplakia we still heavily depend on clinical judgment, adequate biopsy and histopathology for definition of its malignant potential.
\end{abstract}

Keywords: oral leukoplakia, mouth neoplasms, pathology, diagnosis, biopsy.

\section{INTRODUÇÃO}

O câncer de boca é uma denominação que inclui os cânceres de lábio e da cavidade bucal (mucosa bucal, gengivas, palato duro, língua e assoalho da boca). O câncer de lábio é mais frequente em pessoas brancas, e registra maior ocorrência no lábio inferior em relação ao superior. O câncer em outras regiões da boca acomete principalmente tabagistas e os riscos aumentam quando há associação do fumo ao consumo de álcool ${ }^{1}$.

O câncer de boca é o 70 tipo de câncer mais incidente no Brasil. O número de casos novos de câncer de boca estimados para o Brasil, no ano de 2014, foi de 15.290 , destes 11.280 entre homens e de 4.010 nas mulheres. $O$ estado de São Paulo é o que apresenta a maior estimativa de câncer de boca no país (4.500 casos novos), seguido pelo Rio de Janeiro (2.010 casos novos), sendo que os 
estados da Região Norte apresentam a menor estimativa (entre 20 a 190 casos novos). As neoplasias malignas da cavidade bucal apresentam uma incidência maior em homens em relação às mulheres nas regiões Sul e Sudeste do país ${ }^{2}$.

Os fatores que podem levar ao câncer de boca são: idade superior a 40 anos, tabagismo (uso de cachimbos ou cigarros), consumo de álcool, má higiene bucal e uso de próteses dentárias malajustadas, e nos casos dos cânceres de lábio, exposição solar ${ }^{1-4}$. Oitenta por cento dos casos estão associados ao uso de tabaco e álcool ${ }^{5,6}$.

A maioria dos cânceres orais é precedida por lesão visível, embora o tumor possa se desenvolver numa mucosa aparentemente normal ${ }^{6}$.

O método primário de detecção do câncer bucal é o exame clínico da boca, que deve ser realizado anualmente em pacientes acima de 40 anos, o qual é recomendado pela American Cancer Society e pelo Instituto Nacional do Câncer (INCA) no Brasil ${ }^{3,5}$. Cirurgiões dentistas estão numa posição única para examinar pacientes em busca de sinais e sintomas do câncer bucal, pois vêem seus pacientes frequentemente e regularmente quando comparado a outros profissionais de saúde. Embora, a população de maior risco de desenvolver câncer bucal, mais comumente visita médicos do que cirurgiões dentistas ${ }^{5}$. Assim, médicos também precisam ser treinados para realização do exame da cavidade bucal ${ }^{5,7}$.

Diferente das neoplasias malignas da pele, aquelas que ocorrem na cavidade bucal apresentam prognóstico pior, com morbimortalidade elevada, devido a grande vascularização desta área propiciando a disseminação neoplásica, além de o tratamento poder provocar mutilações importantes aos pacientes ${ }^{1}$. A mortalidade resultante do câncer bucal está fortemente correlacionada ao estágio de diagnóstico, prevenção e diagnóstico precoce de lesões precursoras ${ }^{5}$.

O objetivo do presente revisão bibliográfica é abordar os diagnósticos diferenciais anatomopatológicos da leucoplasia bucal, tentando, assim, clarear o conceito desta lesão.

\section{MÉTODOS}

Realizou-se uma revisão bibliográfica narrativa da literatura, com levantamento de artigos científicos sobre leucoplasia bucal até o ano de 2015, sem restrição de idioma. Foram utilizadas as seguintes bases de dados: MEDLINE, PMC (PubMed Central) - NIH (National Institutes of Health), EMBASE (The Excerpta Medica Database), EBSCO e Bireme. Os descritores utilizados foram: leucoplasia, boca, câncer.

\section{REVISÃO DA LITERATURA}

\section{Desenvolvimento do carcinoma espinocelular da cavidade bucal}

O tipo histológico mais comum do câncer de boca é o tipo epidermóide ou espinocelular (CEC) (90\% dos casos), como os da pele e do colo uterino ${ }^{8,9}$.

Como todas as neoplasias epiteliais, acreditasse que o desenvolvimento do CEC da cavidade bucal seja em múltiplas etapas, envolvendo ativação sequencial de oncogenes e inativação de genes supressores tumorais. As etapas de desenvolvimento seriam: mucosa normal, hiperplasia/hiperceratose, displasia leve/moderada, displasia grave/carcinoma in situ e por fim o carcinoma espinocelular invasor ${ }^{9-11}$. Esta progressão em múltiplas etapas permite tanto a detecção precoce do carcinoma espinocelular quanto à de suas lesões precursoras, possibilitando a prevenção do aparecimento desta neoplasia e aumentando a chance de cura dos pacientes ${ }^{10}$.

A Organização Mundial de Saúde (OMS) aceita as seguintes terminologias e definições: 1 . Lesão pré-cancerosa: tecido alterado morfologicamente no qual o câncer ocorre mais comumente do que na sua contraparte aparentemente normal; 2 . Condição pré-cancerosa: estado generalizado associado com um risco significantemente maior de câncer, como por exemplo, fibrose submucosa e líquen plano. Esta última definição significa que o câncer pode surgir em qualquer local da cavidade bucal e não necessariamente numa lesão pré-existente ${ }^{10,12,13}$.

Não se sabe ao certo quantos CEC da cavidade bucal são originados de lesões precursoras e quantos se desenvolvem da mucosa bucal aparentemente normal ${ }^{10,14}$. A literatura mostra que de 0,13 a $18 \%$ das lesões préneoplásicas evoluirão para câncer bucal ${ }^{10}$. As principais lesões pré-cancerosas incluem as leucoplasias e eritroplasias. 


\section{Lesões precursoras do carcinoma espinocelular da cavidade bucal}

Há um consenso geral que leucoplasia e eritroplasia são diagnósticos clínicos sem qualquer conotação com sua histopatologia ${ }^{10}$. O conceito atualmente aceito de leucoplasia é o definido pela Organização Mundial de Saúde (OMS) em 1997: "uma lesão da mucosa bucal predominantemente branca que não pode ser caracterizada como nenhuma outra lesão definível"15. Assim como o conceito atual de eritroplasia, também definido pela OMS em 1997 é "uma placa vermelho fogo que não pode ser caracterizada clinicamente ou patologicamente como nenhuma outra doença definível” ${ }^{\prime 15}$. O Quadro 1 mostra a evolução da definição de leucoplasia e eritroplasia ao longo dos anos.

Quadro 1. Evolução da nomenclatura da leucoplasia e eritroplasia ${ }^{10}$.

\begin{tabular}{|c|c|c|}
\hline & Leucoplasia & Eritroplasia \\
\hline OMS* ${ }^{41}$ & $\begin{array}{c}\text { Uma placa branca que não pode ser } \\
\text { caracterizada clinicamente ou } \\
\text { patologicamente como nenhuma outra } \\
\text { doença. }\end{array}$ & $\begin{array}{c}\text { Placa aveludada e brilhante, a qual } \\
\text { não pode ser caracterizada } \\
\text { clinicamente ou patologicamente } \\
\text { como sendo devido a qualquer outra } \\
\text { condição. }\end{array}$ \\
\hline Axéll et al. ${ }^{42}$ & $\begin{array}{c}\text { Uma placa esbranquiçada que não pode ser } \\
\text { caracterizada clinicamente ou } \\
\text { patologicamente como nenhuma outra } \\
\text { doença e não está associada a nenhum } \\
\text { agente causal físico ou químico, exceto o uso } \\
\text { de tabaco. }\end{array}$ & $\begin{array}{l}\text { Lesões da mucosa bucal que se } \\
\text { apresentam como placas vermelhas } \\
\text { e brilhantes que não pode ser } \\
\text { caracterizada clinicamente ou } \\
\text { patologicamente como qualquer } \\
\text { outra condição. }\end{array}$ \\
\hline Axéll et al. ${ }^{21}$ & $\begin{array}{l}\text { Uma lesão predominantemente branca da } \\
\text { mucosa bucal que não pode ser } \\
\text { caracterizada clinicamente ou } \\
\text { patologicamente como nenhuma outra lesão } \\
\text { definível; algumas leucoplasias podem se } \\
\text { transformar em câncer. }\end{array}$ & $\begin{array}{l}\text { Lesões da mucosa bucal que se } \\
\text { apresentam como áreas vermelhas e } \\
\text { não podem ser diagnosticadas como } \\
\text { nenhuma outra lesão definível. }\end{array}$ \\
\hline $\begin{array}{l}\text { Pindborg et } \\
\text { al. }{ }^{15} \text { (OMS*) }\end{array}$ & $\begin{array}{c}\text { Uma lesão predominantemente branca da } \\
\text { mucosa bucal que não pode ser } \\
\text { caracterizada como nenhuma outra lesão } \\
\text { definível. }\end{array}$ & $\begin{array}{c}\text { Uma placa vermelho fogo que não } \\
\text { pode ser caracterizada clinicamente } \\
\text { ou patologicamente como nenhuma } \\
\text { outra doença definível. }\end{array}$ \\
\hline
\end{tabular}

*OMS: Organização Mundial de Saúde.

\section{Leucoplasia bucal}

A leucoplasia bucal é a lesão precursora dos CEC mais bem conhecida. Estudos têm mostrado que 16 a $62 \%$ dos carcinomas orais estão associados a lesões leucoplásicas no momento do diagnóstico ${ }^{10}$. 0 risco de desenvolver câncer é 8 a 10 vezes maior em pacientes que apresentam leucoplasia do que naqueles que não apresentam ${ }^{16}$. O tempo de evolução para câncer pode variar de 3 meses a 10 anos $^{17}$.

A leucoplasia bucal é mais frequente em homens idosos e sua frequência aumenta com o avanço da idade. Estima-se que menos de $1 \%$ dos homens afetados tenham menos de 30 anos e a prevalência aumenta para $8 \%$ em homens acima de 70 anos e $2 \%$ em mulheres acima de 70 anos $^{18}$.

Esta lesão tem uma taxa anual de malignização que varia de 0,1 a $17 \%{ }^{18}$. Alguns fatores podem contribuir para o aumento de chance de transformação maligna nas leucoplasias ${ }^{18-20}$ :

- Sexo: mulheres tendem a ter um risco maior de malignização;

- Leucoplasia bucal de longa data;

- Locais de maior risco: assoalho da boca, região ventrolateral da língua e palato mole;

- Leucoplasias em não fumantes; 
- Leucoplasias do tipo não homogêneo; e

- Presença de displasia epitelial moderada ou severa.

As leucoplasias podem ser divididas nos tipos: homogêneo e não homogêneo. A homogênea se apresenta como uma placa ou mancha predominantemente branca, uniforme, plana, fina, cuja superfície pode exibir sulcos rasos, ser lisa ou levemente enrugada. A não homogênea apresenta-se como uma placa ou mancha branca exibindo superfície irregular, nodular ou exofítica, além de poder apresentar-se como uma placa ou mancha vermelha e branca (eritroleucoplasia) ${ }^{21}$. 0 tipo de leucoplasia tem influência sobre o prognóstico, pois a leucoplasia não homogênea contém componentes eritematosos, nodulares e/ou verrucosos, os quais apresentam um alto potencial maligno quando comparados com as leucoplasias homogêneas ${ }^{10}$.

O risco de desenvolvimento de câncer é 4 a 5 vezes maior na leucoplasia não homogênea do que na homogênea ${ }^{10}$. A taxa de transformação maligna em leucoplasia verrucosa é maior que $70,3 \%{ }^{17}$. A taxa de transformação maligna na leucoplasia homogênea é de $5 \%$, mostrando que mesmo este tipo requer acompanhamento ${ }^{10,19}$.

Qualquer local da mucosa bucal pode ser acometido e a leucoplasia pode se apresentar como lesão única ou lesões múltiplas, bem ou mal delimitadas. $O$ assoalho da boca e ventre de língua são os locais com maior predisposição à transformação maligna ${ }^{22}$.

Um diagnóstico estabelecido para uma lesão branca ou predominantemente branca exclui o diagnóstico de leucoplasia. Entretanto, o uso de tabaco e infecção por Candida são frequentemente mencionados como fatores etiológicos para leucoplasia, e ambos estão relacionados ao prognóstico ${ }^{10}$. Os principais fatores de risco para o desenvolvimento de leucoplasia são o uso de tabaco e álcool, radiação ultravioleta e microorganismos como o HPV, principalmente subtipos 16 e $18^{22,23}$.

Leucoplasias associadas ao hábito de fumar parecem ter um potencial maligno menor do que aquelas não relacionadas ao tabagismo. Além disto, há uma forte relação entre tabagismo e desenvolvimento de leucoplasia no assoalho da boca, enquanto nos não fumantes esta lesão é mais comum na borda da língua ${ }^{10}$.
A associação de infecção por Candida e risco para desenvolvimento de câncer originou dos achados de associação desta com leucoplasia não homogênea. O principal tipo de Candida isolada das leucoplasias não homogêneas é a Candida albicans, algumas das quais têm um alto potencial de formação de nitrosaminas, sugerindo a produção endógena de nitrosaminas carcinogênicas $^{10}$.

Além desses fatores, a possível implicação do papiloma vírus humano (HPV) na etiologia e potencial transformação maligna de lesões prémalignas da cavidade bucal tem sido estudada extensivamente $^{4,24}$. O HPV-16 é o subtipo de HPV mais frequentemente associado ao CEC bucal, e ambos HPV-16 e 18 têm um papel importante na transformação maligna. A taxa de detecção de HPV em CEC bucal varia de 0 a $94 \%$ e em alguns estudos o HPV tem sido detectado também em alta frequência na mucosa normal, leucoplasia benigna e lesão intraepitelial ${ }^{24}$.

\section{Diagnóstico de leucoplasia bucal}

Para o diagnóstico de leucoplasia, lesões que pertencem a outras entidades devem ser excluídas, tais como líquen plano, lupus eritematoso, leucoedema, nevo esponjoso branco e lesões para as quais a etiologia pode ser definida, como queratose friccional, lesões de mordedura e de contato e o palato do fumante ${ }^{10}$.

O diagnóstico diferencial da leucoplasia bucal se faz com lesões brancas na mucosa que não podem ser destacadas a raspagem. Os principais diagnósticos diferenciais são ${ }^{25}$ :

- Leucoedema, que ocorre mais frequentemente em negros e está relacionado ao tabagismo. Apresentase como uma película de superfície enrugada distorcida, branco-cinzenta, que se estende através da mucosa bucal. Histologicamente caracterizada por edema acentuado das células da camada espinhosa, podendo ter vários graus de hiperparaqueratose. Ao contrário de leucoplasia, que geralmente apresenta uma cor branca mais definida, o eucoedema torna-se menos visível ou desaparece ao se apertar o tecido;

- Leucoplasia pilosa é caracterizada por uma lesão branca de superfície rugosa 
que não é removível por raspagem, preferencialmente localizada na extremidade da língua bilateralmente. O diagnóstico exige a demonstração da presença do vírus Epstein-Barr na lesão;

- Líquen plano, que se apresenta como uma placa branca e solitária. É comum que a placa do Líquen plano tenha bordas com limites imprecisos e, além disso, tem o padrão reticular típico, o que ajuda no diagnóstico diferencial com a leucoplasia. Há casos onde coexistem as duas entidades;

- Lesões orais liquenóides que estão relacionados ao amálgama de restaurações. Podem aparecer na gengiva, língua ou na mucosa bucal, nas imediações de restaurações metálicas, quer como uma placa ceratótica como a leucoplasia ou imitando o líquen plano. Em alguns dos casos, a remoção das restaurações de metal ocasionam a sua regressão;

- Lúpus eritematoso apresenta lesões discóides orais, as quais são comuns em indivíduos com lesões de pele de lúpus discóide e naqueles com lúpus eritematoso sistêmico, podendo ser a primeira manifestação destes. Algumas lesões orais discóides podem ser muito parecidas com a leucoplasia e o líquen plano, mas, muitas vezes têm uma maior componente eritematoso e tem um arranjo radial dos capilares característico na periferia da placa.

- Nevo branco esponjoso é uma doença rara, hereditária autossômica dominante. Apresenta-se como lesão branca, de superfície rugosa, afetando vários pontos da mucosa. Pode estar presente ao nascimento ou tornar-se evidente na puberdade. Muitas vezes afeta a mucosa oral de forma simétrica. Microscopicamente, o epitélio é espessado com acentuada espongiose, acantose e paraceratose. São lesões assintomáticas, sem nenhuma evidência de uma possível transformação maligna.
Como o diagnóstico de leucoplasia é feito por exclusão de outra doença ou desordem conhecida, a biópsia em lesões leucoplásicas é mandatória ${ }^{10,26,27}$. Alguns autores recomendam que o diagnóstico seja confirmado histologicamente se a lesão persistir por 2 a 4 semanas ou quando outros fatores etiológicos tenham sido descartados ${ }^{20,28}$. Além disso, nenhum achado clínico é mais ou menos propenso a carcinoma, por isso também a necessidade de biópsia ${ }^{26}$.

O corante azul de toluidina é uma possibilidade para ajudar nos casos de leucoplasia oral, direcionando áreas mais suspeitas de malignidade para biópsia. Os resultados do estudo de Gandolfo et al. ${ }^{29}$ sugerem que a coloração azul escura significa um teste positivo e áreas densamente escuras estão mais associadas a malignidade a histologia do que áreas azuis claras que estão mais associadas à benignidade. Porém, a coloração de azul de toluidina pode apresentar um falso-positivo em condições de trauma ou inflamação, sendo indicada uma nova coloração após duas semanas, quando o risco de falsopositivo cai para $10 \%{ }^{30}$.

Microscopicamente, a leucoplasia pode ser caracterizada por uma gama de alterações na renovação e maturação epitelial, tais como hiperplasia de células escamosas (hiperceratose, acantose, hiperceratose associada à acantose), displasia epitelial, Carcinoma in situ e CEC invasor $^{20,27}$.

A hiperplasia de células escamosas descreve um aumento no número de células. Esta pode ser da camada espinhosa (acantose) e/ou da camada basal/parabasal (hiperplasia de células basais) A arquitetura mostra estratificação regular sem atipias ${ }^{8}$. Displasia epitelial é definida como uma lesão pré-cancerosa do epitélio escamoso estratificado, caracterizada por atipias celulares e perda maturação e estraficação normal deste. Carcinoma espinocelular in situ é definido como uma lesão na qual toda a espessura ou quase toda espessura do epitélio escamoso mostra alterações de diferenciação e maturação, porém sem ultrapassar a membrana basal do epitélio ${ }^{8,10}$. CEC invasor é uma neoplasia epitelial com variados graus de diferenciação escamosa e propensão precoce e extensa de metástase para linfonodos ${ }^{8}$. 
Displasia epitelial

Segundo a OMS, o diagnóstico de displasia epitelial é firmado quando pelo menos duas alterações arquiteturais e duas citológicas estiverem presentes no epitélio. As alterações arquiteturais são: estratificação epitelial irregular, perda de polaridade das células basais, cristas epidérmicas "em gota", aumento do número de figuras de mitose, mitoses anormais na superfície, disceratose e pérolas córneas. As alterações citológicas (ou atipias citológicas) são: variação anormal no tamanho dos núcleos e das células, pleomorfismo celular, aumento da relação núcleocitoplasma, figuras de mitose atípicas, aumento no número e tamanho dos nucléolos e hipercromasia nuclear $^{8,10,27}$.

A displasia é um espectro, dividido em leve, moderada e severa, dependendo da espessura do epitélio comprometida pelas alterações ${ }^{9}$. A displasia leve é uma alteração arquitetural limitada ao terço inferior do epitélio. Na displasia moderada a alteração se estende para o terço médio. A displasia severa compromete mais do que dois terços do epitélio, com maior grau de atipia ${ }^{8}$. A extensão de comprometimento do epitélio está diretamente relacionada ao risco de risco de malignização destas lesões ${ }^{9}$.
Estudos têm mostrado a grande variação inter e intra-examinador no acesso a presença ou ausência e graduação da displasia do epitélio bucal. O Working Group da OMS sugere duas categorias de classificação para melhoria na concordância entre patologistas: 1 . displasia ausente ou questionável ou leve: consideradas de baixo risco de malignização; 2 . displasia moderada ou severa: consideradas de alto risco de malignização ${ }^{31}$. Assim podemos dividir as displasias em displasias de baixo risco e displasias de alto risco, sendo que as últimas têm um risco 4,57 vezes maior de transformação maligna, quando comparadas às de baixo risco, durante os primeiros 2 a 3 anos de seguimento ${ }^{32}$.

De acordo com a sugestão da OMS desde 2005, este esquema tradicional de graduação da displasia continua sendo usado. Em adição, o conceito de neoplasia intraepitelial escamosa foi introduzido com a graduação de I a III $^{8,33}$. A Classificação de Ljubljana foi originalmente descrita para graduação de lesões precursoras do epitélio laríngeo, mas também tem sido usada para lesões precursoras de câncer da cavidade bucal ${ }^{33}$. O Quadro 2 compara os três sistemas de classificação.

Quadro 2. Esquemas de classificação que histologicamente categorizam as lesões precursoras da cavidade bucal ${ }^{8}$.

\begin{tabular}{|c|c|c|}
\hline Classificação da OMS, 2005. & $\begin{array}{l}\text { Neoplasia intraepitelial } \\
\left.\text { escamosa (NIE/SIN }{ }^{*}\right)\end{array}$ & $\begin{array}{c}\text { Classificação de Ljubljana - } \\
\text { Lesão intraepitelial escamosa } \\
\text { (LIE/SIL") }\end{array}$ \\
\hline $\begin{array}{c}\text { Hiperplasia de células } \\
\text { escamosas }\end{array}$ & --- & $\begin{array}{c}\text { Hiperplasia (simples de células } \\
\text { escamosas) }\end{array}$ \\
\hline Displasia leve & SIN 1 & $\begin{array}{c}\text { Hiperplasia de células } \\
\text { basais/parabasais }\end{array}$ \\
\hline Displasia moderada & SIN 2 & \multirow{2}{*}{ Hiperplasia atípica ${ }^{\S}$} \\
\hline Displasia severa & \multirow{2}{*}{ SIN 3} & \\
\hline Carcinoma in situ & & Carcinoma in situ \\
\hline
\end{tabular}

*NIE/SIN: neoplasia intraepitelial escamosa / squamous intraepithelial neoplasia. "LIE/SIL: lesão intraepitelial escamosa / squamous intraepithelial lesion. ${ }^{\S}$ : chamado de epitélio de risco.

Lesões com displasia epitelial mais frequentemente evoluem para carcinoma do que aquelas sem displasia. Além disso, achados de displasia epitelial são comumente vistos no epitélio adjacente a carcinomas orais. Entretanto, displasias epiteliais não necessariamente evoluem para carcinoma e podem até mesmo regredir ${ }^{10}$.

Colloq Vitae 2016 mai-ago; 8(2):37-45. DOI: 10.5747/cv.2016.v08.n2.v166

\section{Displasia epitelial e leucoplasia bucal}

A displasia bucal não está associada a nenhuma aparência clínica específica. Entretanto leucoplasia e eritroplasia são as lesões classicamente associadas com alterações displásicas ${ }^{10}$. A frequência de displasia em leucoplasia varia de menos de $1 \%$ a $30 \%$. A eritroplasia, uma lesão menos frequente do que a 
leucoplasia, quase invariavelmente apresenta displasia ou carcinoma franco, histologicamente ${ }^{10}$.

Displasia epitelial pode ser observada em biópsias de leucoplasias homogêneas, mas é mais comumente diagnosticada em leucoplasias não homogêneas ${ }^{10}$.

As leucoplasias podem ser classificadas histologicamente em: leucoplasias displásicas ou não displásicas ${ }^{27}$. Tem sido relatada a ocorrência de transformação maligna em 3\% das lesões com displasias leve, em $4 \%$ das com displasia moderada e em $43 \%$ daquelas com displasia severa ${ }^{10}$. Transformação carcinomatosa também pode ser observada em leucoplasias não displásicas, e alterações genéticas, particularmente no cromossomo 3, têm sido observadas na maioria das lesões não displásicas e queratóticas ${ }^{27}$.

No estudo de Waldron e Shafer ${ }^{34}$, com avaliação de 3256 leucoplasias orais, foram observadas alterações não displásicas em 80,1\% dos casos e uma maior frequência de displasia em mulheres.

\section{Tratamento da leucoplasia bucal}

O tratamento da leucoplasia é baseado na premissa da deteç̧ão precoce e manejo ativo dos pacientes para prevenir o desenvolvimento de $\mathrm{CEC}^{35}$. Os protocolos de manejo desta entidade clínica estão direcionados a redução de risco de transformação maligna. Os mais frequentemente usados são: observação e seguimento da lesão, modificação do hábito de ingestão de bebidas alcoólicas e de fumar dos pacientes, quimioproteção (retinóides e carotenóides), extirpação (cirurgia convencional, eletrocauterização, ablação com laser ou crioterapia), terapia fotodinâmica e aplicação tópica de bleomicina e 5-fluoracil ${ }^{18,35,36}$.

Apesar do tratamento escolhido, cerca de $6,2 \%$ das leucoplasias se transformarão em câncer ${ }^{37}$.

$\mathrm{Na}$ presença de displasia epitelial moderada ou severa, o tratamento cirúrgico está recomendado. Leucoplasias apresentando risco baixo ou moderado de malignização podem ser completamente removidas ou não, e a decisão deve considerar outros fatores, tais como localização e tamanho da lesão, no caso de fumantes, o engajamento de pacientes na tentativa de parar de fumar. A recorrência da leucoplasia após a cirurgia está relatada em 10 a 35\% dos casos descritos ${ }^{18}$ e desenvolvimento de câncer em 3 a $9 \%$ 10. A recomendação atual da OMS, porém, é ressecção total ou ablação com laser de qualquer leucoplasia ou eritroplasia da região bucal e orofaríngea, quando possível, independente da presença ou não de displasia ${ }^{38}$. 0 tratamento não cirúrgico pode ser considerado, especialmente em pacientes com grande área da mucosa bucal comprometida ou naqueles com problemas médicos, o que consequentemente eleva o risco cirúrgico ${ }^{18}$.

O estudo de Nagaraju et al. ${ }^{39}$ mostrou que a combinação de Lugol e azul de Toluidina tem sensibilidade de $92,7 \%$ em detectar displasia ou lesões malignas e especificidade de $60 \%$. O estudo de Su et al. ${ }^{40}$, com 7.975 pacientes, mostrou que o azul de Toluidina é uma boa ferramenta para o rastreamento de leucoplasias em indivíduos com alto risco de desenvolver câncer de boca, como aqueles que fumam cigarro ou mascam betel, podendo detectar mais casos do que somente o rastreio visual. Assim, estas colorações podem ser rotineiramente utilizadas como um método para auxiliar na escolha do local da biópsia e no seguimento de lesões pré-malignas ${ }^{39,40}$.

O desafio no campo do pré-câncer bucal é predizer quais lesões eventualmente irão evoluir para carcinoma ${ }^{10}$. Alguns estudos têm tentado elucidar os determinantes moleculares e as viaschaves de sinalização envolvidas na transformação de lesões pré-malignas em câncer e assim então identificar novos alvos diagnósticos e terapêuticos ${ }^{16}$. Os três principais biomarcadores associados com alto risco de câncer em leucoplasia são: alta polissomia cromossomal, grande acúmulo de proteína p53 na camada parabasal e perda da heterozigosidade em $3 p$ ou $9 p{ }^{17}$.

Apesar das modernas técnicas de biologia molecular e imunoistoquímica, as características clínicas convencionais (tipo de leucoplasia) e a histopatologia (presença ou não de displasia epitelial) ainda são os parâmetros mais importantes para predizer a transformação maligna em lesões pré-malignas da cavidade bucal na rotina diagnóstica em patologia bucal ${ }^{5,10,37}$.

O correto diagnóstico das lesões da cavidade bucal permite um tratamento adequado e eficaz, pois poderá ser iniciado o mais breve possível, com possibilidade maior de chance de cura e menor morbidade aos pacientes. No caso das leucoplasias bucais ainda dependemos 
fortemente do julgamento clínico, biópsias adequadas e do exame histopatológico para definição do seu potencial de malignização.

\section{Conflito de interesse}

Os autores declaram não haver qualquer potencial conflito de interesse que possa interferir na imparcialidade deste trabalho científico.

\section{REFERÊNCIAS}

1. Brasil. Ministério da Saúde. Secretaria de Atenção a Saúde. Instituto Nacional do Câncer. Estimativa 2010: Incidência de câncer no Brasil. Rio de Janeiro: INCA, 2009.

2. Brasil. Ministério da Saúde. Secretaria de Atenção a Saúde. Instituto Nacional do Câncer. Estimativa 2014: Incidência de câncer no Brasil. Rio de Janeiro: INCA, 2014. [acesso em 16 fev 2016]. Disponível

em: http://www.inca.gov.br/estimativa/2014/estimativ a-24042014.pdf

3. Instituto Nacional do Câncer (INCA). Câncer de Boca. [acesso em 5 fev 2016] .Disponível em: http://www.inca.gov.br/

4. Sciubba JJ. Oral cancer. The importance of early diagnosis and treatment. Am J Clin Dermatol. 2001;2(4):239-51.

5. Applebaum E, Ruhlen TN, Kronenberg FR, Hayes C, Peters ES. Oral Cancer Knowledge, Attitudes and Practices: A Survey of Dentists and Primary Care Physicians in Massachusetts. J Am Dent Assoc 2009;140: 461-7.

6. Hande $A H$, Chaudhary MS. Cytomorphometric analysis of buccal mucosa of tobacco chewers. Romanian J Morphol Embryol. 2010, 51(3):527-32.

7. Bornstein MM, Lüönd-Valeskeviciute I, Altermatt HJ, Stauffer E, Buser D. Oral mucosal lesions diagnosed in a stomatology service. An examination of clinico-pathological findings from the year 2003. Schweiz Monatsschr Zahnmed. 2006;116(5):468-75.

8. Barnes L, Eveson JW, Reichart P, Sindransky D (Eds.) World Health Organization Classification of Tumours. Pathology and genetics of head and neck tumours. IARC Press: Lyon; 2005.

9. Lingen MW, Kumar V. Cavidade oral. Cabeça e Pescoço. In: Kumar, V, Abbas AK, Fausto N (Eds.). Robbins e Cotran, Bases Patológicas das Doenças. 7.ed. Capítulo 16, pp. 814-823. Rio de Janeiro: Editora Elsevier; 2005.

10. Reibel J. Prognosis of Oral Pre-malignant
Lesions: Significance of Clinical, Histopathological, and Molecular Biological Characteristics. CROBM 2003;14:47-62.

DOI: http://dx.doi.org/10.1177/154411130301400105

11. Mehrotra R, Gupta A, Singh M, Ibrahim R. Application of cytology and molecular biology in diagnosing premalignant or malignant oral lesions. Mol Cancer. 2006;5:11 DOI: http://dx.doi.org/10.1186/1476-4598-5-11

12. Ben Slama L. Precancerous lesions of the buccal mucosa. Rev Stomatol Chir Maxillofac. 2001;102(2):77-108.

13. Warnakulasuriya $S$, Johnson NW, van der Waal I. Nomenclature and classification of potentially malignant disorders of the oral mucosa. J Oral Pathol Med. 2007;36(10):575-80.

14. Hsue SS, Wang WC, Chen CH, Lin CC, Chen YK, Lin LM. Malignant transformation in 1458 patients with potentially malignant oral mucosal disorders: a follow-up study based in a Taiwanese hospital. J Oral Pathol Med. 2007;36(1):25-9.

15. Pindborg JJ, Reichart $P$, Smith CJ, van der Waal I. World Health Organization: histological typing of cancer and precancer of the oral mucosa. Berlin: Springer-Verlag; 1997.

16. Wang Z, Feng $X$, Liu X, Jiang L, Zeng X, Ji N, Li J, Li L, Chen Q. Involvement of potential pathways in malignant transformation from Oral Leukoplakia to Oral Squamous Cell Carcinoma revealed by proteomic analysis. BMC Genomics 2009. 10:383. DOI: http://dx.doi.org/10.1186/1471-2164-10-383

17. Lee JJ, Hong WK, Hittelman WN, Mao L, Lotan $\mathrm{R}$, Shin DM, Benner SE, $\mathrm{Xu} X-\mathrm{C}$, Lee JS, Papadimitrakopoulou VM, Geyer C, Perez C, Martin JW, El-Naggar AK, Lippman SM. Predicting Cancer Development in Oral Leukoplakia: Ten Years of Translational Research. Clin Cancer Res 2000;6:1702-1710.

18. Ribeiro AS, Salles PR, Silva TA, Mesquita RA. A Review of the Nonsurgical Treatment of Oral Leukoplakia. Int J Dent. 2010; Article ID 186018, 10 pages.

DOI:

http://dx.doi.org/10.1155/2010/186018

19. Napier SS, Speight PM. Natural history of potentially malignant oral lesions and conditions: an overview of the literature. J Oral Pathol Med. 2008;37(1):1-10.

20. Vázquez-Álvarez $R$, Fernández-González $F$, Gándara-Vila P, Reboiras-López D, García-García A, Gándara-Rey JM. Correlation between clinical and pathologic diagnosis in oral leukoplakia in 54 
patients. Med Oral Patol Oral Cir Bucal. 2010;15(6):e832-8.

21. Axéll T, Pindborg JJ, Smith CJ, van der Waal I. Oral white lesions with special reference to precancerous and tobacco-related lesions: conclusions of an international symposium held in Uppsala, Sweden, May 18-211994. International Collaborative Group on Oral White Lesions. J Oral Pathol Med. 1996;25(2):49-54.

22. Longshore SJ, Camisa C. Detection and management of premalignant oral leukoplakia. Dermatol Ther. 2002;15(3):229-35.

23. Dietrich T, Reichart PA, Scheifele C. Clinical risk factors of oral leukoplakia in a representative sample of the US population. Oral Oncol. 2004;40(2):158-63.

24. Khovidhunkit SP, Buajeeb W, Sanguansin S, Poomsawat S, Weerapradist W. Detection of Human Papillomavirus in Oral Squamous Cell Carcinoma, Leukoplakia and Lichen Planus in Thai Patients. Asian Pacific J Cancer Prev. 2008;9:771-5.

25. Martínez-Sahuquillo Márquez A, Gallardo Castillo I, Cobos Fuentes MJ, Caballero Aguilar J, Bullón Fernández P. La leucoplasia oral. Su implicación como lesión precancerosa. Av Odontoestomatol. 2008;24(1):33-44.

26. Lee JJ, Hung HC, Cheng SJ, Chen YJ, Chiang CP, Liu BY, Jeng JH, Chang HH, Kuo YS, Lan WH, Kok SH. Carcinoma and dysplasia in oral leukoplakias in Taiwan: prevalence and risk factors. Oral Surg Oral Med Oral Pathol Oral Radiol Endod. 2006;101(4):472-80.

27. Hildebrand L, Carrard V, Lauxen I, Quadros O, Chaves A, Sant'Ana-Filho M. Evaluation of cell proliferation rate in non-dysplastic leukoplakias. Med Oral Patol Oral Cir Bucal. 2010;15(2):e328-34.

28. Huber MA. A review of premalignant oral conditions. Tex Dent J. 2006;123(6):502-9.

29. Gandolfo S, Pentenero M, Broccoletti R, Pagano $M$, Carrozzo M, Scully C. Toluidine blue uptake in potentially malignant oral lesions in vivo: clinical and histological assessment. Oral Oncol. 2006;42(1):89-95.

30. Bsoul SA, Huber MA, Terezhalmy GT. Squamous cell carcinoma of the oral tissues: a comprehensive review for oral healthcare providers. J Contemp Dent Pract. 2005;6(4):1-16.

31. Warnakulasuriya S, Reibel J, Bouquot J, Dabelsteen E. Oral epithelial dysplasia classification systems: predictive value, utility, weaknesses and scope for improvement. J Oral Pathol Med.
2008;37(3):127-33.

32. Liu W, Wang YF, Zhou HW, Shi P, Zhou ZT, Tang

GY. Malignant transformation of oral leukoplakia: a retrospective cohort study of 218 Chinese patients. BMC Cancer. 2010;10(1):685.

33. Driemel O, Hertel K, Reichert TE, Kosmehl H. Current classification of precursor lesions of oral squamous cell carcinoma principles of the WHO classification 2005. Mund Kiefer Gesichtschir. 2006;10(2):89-93.

34. Waldron CA, Shafer WG. Leukoplakia revisited. A clinicopathologic study 3256 oral leukoplakias. Cancer. 1975;36(4):1386-92.

35. López-Jornet $P$, Camacho-Alonso F. The quality of internet information relating to oral leukoplakia. Med Oral Patol Oral Cir Bucal. 2010;15(5):e727-31.

36. Lodi G, Sardella A, Bez C, Demarosi F, Carrassi A. Interventions for treating oral leukoplakia. Cochrane Database Syst Rev. 2004;(3):CD001829.

37. Dunsche A, Härle F. Precancer stages of the oral mucosa: a review. Laryngorhinootologie. 2000;79(7):423-7.

38. Van der Waal I. Potentially malignant disorders of the oral and oropharyngeal mucosa; terminology, classification and present concepts of management. Oral Oncol. 2009;45(4-5):317-23.

39. Nagaraju K, Prasad S, Ashok L. Diagnostic efficiency of toluidine blue with Lugol's iodine in oral premalignant and malignant lesions. Indian J Dent Res. 2010;21(2):218-23.

40. Su WW, Yen AM, Chiu SY, Chen TH. Does toluidine blue detect more oral cancer? J Dent Res. 2010;89:933-7.

41. WHO. Collaborating Centre for Oral Precancerous Lesions. Definition of leukoplakia and related lesions: an aid to studies on oral precancer. Oral Surg Oral Med Oral Pathol Oral Radiol Endod. 1978; 46:518-39.

42. Axéll T, Holmstrup P, Kramer IRH, Pindborg JJ, Shear M. International seminar on oral leukoplakia and associated lesions related to tobacco habits. Community Dent Oral Epidemiol. 1984; 12:145-54.

Recebido para publicação em 03/08/2015

Revisado em 03/06/2016

Aceito em 17/06/2016 